\title{
Ultrastructural Findings, at Micrometric and Nanometric Scales, in Rectal and Muscular Mucosa of Patients with HIV/AIDS and Anorectal Pathology
}

\author{
Annunziato Maria Antonieta ${ }^{*}$, Sardiñas Carlos ${ }^{2}$, Finol Hector ${ }^{3}$, Carvajal Ana ${ }^{4}$, \\ González Roschman ${ }^{3}$, De Gouveia Yetsenia ${ }^{5}$, García Estefanie ${ }^{5}$, Garibaldi Liseth ${ }^{6}$
}

\begin{abstract}
${ }^{1}$ Department of Internal Medicine, Medical Attention Center, General Checkups, Medical Exams and Consultations, Wheelchair Center (BANDESIR), Caracas, Venezuela

${ }^{2}$ Coloproctology Unit, Caracas University Hospital (HUC), Caracas, Venezuela

${ }^{3}$ Center for Electron Microscopy, Sciences Faculty, Central University of Venezuela, Caracas, Venezuela

${ }^{4}$ Department of Infectious Diseases, University Hospital of Caracas (HUC), Caracas, Venezuela

${ }^{5}$ School of Biology, Faculty of Sciences, Central University of Venezuela, Caracas, Venezuela

${ }^{6}$ Research Assistant Department of Electronic Microscopy, Venezuelan Institute of Scientific Research (IVIC), Altos de Pipe,

Venezuela
\end{abstract}

Email: *Annunziato.maria@gmail.com

How to cite this paper: Antonieta, A.M., Carlos, S., Hector, F., Ana, C., Roschman, G., Yetsenia, D.G., Estefanie, G. and Liseth, G. (2018) Ultrastructural Findings, at Micrometric and Nanometric Scales, in Rectal and Muscular Mucosa of Patients with HIV/AIDS and Anorectal Pathology. International Journal of Clinical Medicine, 9 , 481-493.

https://doi.org/10.4236/ijcm.2018.95041

Received: April 17, 2018

Accepted: May 28, 2018

Published: May 31, 2018

Copyright ( $) 2018$ by authors and Scientific Research Publishing Inc. This work is licensed under the Creative Commons Attribution International License (CC BY 4.0).

http://creativecommons.org/licenses/by/4.0/ c) (i) Open Access

\begin{abstract}
Objective: To determine the ultrastructural findings on Rectal Mucosa (RM) of patients with HIV/AIDS and anorectal pathologies (ARP), at micrometric and nanometric scales. Materials and methods: 5 patients were evaluated, 18 - 55 years old, with ARP (HIV co-infection with HPV, $\mathrm{n}=4$, and HIV-negative patient with HPV infection) (control $n=1$ ), who were referred to the Coloproctology Unit of the HUC, and subjected to rectoscopy and biopsy. RM samples were identified, placed in a sterile plastic bottle with $1 \mathrm{~mL}$ of $2 \%$ glutaraldehyde and immediately transported for routine processing of fine cut $(60-90 \mathrm{~nm})$ to be evaluated via Transmission Electron Microscopy (TEM). They were fixed with Karnovsky solution with Millonig phosphate buffer ( $\mathrm{pH} 7.4$ and $320 \mathrm{mOsm}$ ) and post-fixed with $\mathrm{OsO}_{4}$ under the same conditions of $\mathrm{pH}$ and osmolarity. Results: Ultrastructural findings, at $10^{-6}$ scale: 1) Intestinal mucosa: vacuoles of mucus of different sizes that seem to be fused. 2) Smooth muscle cells: loss of definition of contractile myofilaments mass. 3) Unmyelinated axons and terminals of Schwann cells (SC): Edema and loss of their plasma membranes in some areas of association with axon terminals as well as abundant collagen fibers associated with SC. Ultrastructural findings, at $10^{-9}$ scale: 1) Smooth muscle cells: folded wrapper cores
\end{abstract}


and edema of mitochondria and rough endoplasmic reticulum cisterns (RER). 2) Myelinated axon terminals: Loss of synaptic vesicles. 3) Fibroblasts: One observes mitochondria and cisterns of RER with alterations. All these alterations can generate intestinal and anorectal dysfunction in these patients. Conclusions: The HIV causes changes in rectal and muscular mucosa despite HAART treatment with undetectable viral load.

\section{Keywords}

Anorectal Pathologies, HIV/AIDS, Transmission Electron Microscopy (TEM), Nanometric Scales, Rectal Mucosa, Caveolae, Endothelial Cell, Nano-Drugs

\section{Introduction}

Gastrointestinal pathologies/disorders (GI) are common during the course of HIV infection. 35\% of patients have GI symptoms as clinical presentation of HIV. These are usually very varied and include weight loss, dysphagia, anorexia, gastric disorders and diarrhea, the latter being one of the most common symptoms occurring in $30 \%$ to $50 \%$ of patients in North America and Europe [1] [2]. Affectation that produces HIV along the digestive tract may compromise the mouth, esophagus, stomach, small intestine, colon and terminal portions as anus and rectum [3] [4] [5]. Among the most frequent anorectal diseases, the following have been reported: condyloma acuminata (human papilloma virus [HPV]), fistulas, perianal abscesses, hemorrhoids, fissures, perianal herpes, nonspecific proctitis, rectal masses (polyps, diverticula, Kaposi's sarcoma). Opportunistic and neoplastic anal and rectal infections have also been described in these patients, with a variety of symptoms that can range from anorectal pain, tenesmus and discharge from the rectum [6] [7] [8].

The intestinal mucosa (IM) plays an important role in the pathogenesis as well as a persistent HIV area despite the fact that Highly Active Antiretroviral Therapy (HAART) is effective [9] [10]. The rectal mucosa (RM) is the main gateway into groups of men who have sex with men (MSM). The virus can penetrate by direct trauma of the RM, after which it has access to the microcirculation, however, even without existing trauma, HIV can penetrate the RM by viral absorption through specialized epithelial cells called M present in the epithelium associated follicles [10], by transcytosis across epithelial cells or by direct sampling of dendritic cells from the lamina propia. HIV temporarily may open tight junctions between epithelial cells generating a gradient that drives viral migration of dendritic cells through R5 receptor [11]. In addition, the IM contains a high percentage of cells that are the main target of HIV and represents the ideal site of viral replication and depletion of CD4+ T cells [12] [13]. Previous research has shown that viral replication remains in lymphoid tissue for at least two years despite the fact that HAART has achieved complete suppression of viral load in peripheral blood. This also demonstrates incomplete suppression of 
viral replication as well as the increased activation of the immune system and persistent intestinal inflammation at the level of the IM [14]. It is relevant to notice an important finding which is the existence of efflux pumps that are expressed in the intestinal epithelium called P glycoprotein-1 (P-gp), which eject different substances outside the cells and carrying various substrates across the cell membrane including antiretroviral drugs as protease inhibitors (PI) and non-nucleoside inhibitors of reverse transcriptase (NNIRT). Increased expression of intestinal P-gp may reduce the absorption of drugs that are substrates of P-gp and may result in reduced bioavailability and subtherapeutic plasma levels [15].

Transmission Electron microscopy studies have detected the presence of the virus and changes in the lymphoid composition in IM with depletion of T lymphocytes [16] [17]. GI disorders caused by HIV are due in part to the presence of opportunistic pathogens or hidden enteric infections, due to indirect effects of HIV on the intestinal epithelium or to indirect secondary effects of immunological abnormalities in the absence of enteric pathogens unidentifiable (e.g.: lysing infected cells expressing viral proteins on their surface); as well as injury or damage of the intestinal mucosa by direct cytopathic effect [17] [18] [19].

In this specific research, we evaluate the ultrastructural findings, at micrometric and nanometric scales, in rectal and muscular mucosa of patients with HIV/AIDS and anorectal pathologies in order to achieve a better understanding of this pathology and a therapeutic directed to target with perspectives to upgrade the quality of life.

Objective: To determine the ultrastructural findings on RM of patients with HIV/AIDS and ARP, at micrometric and nanometric scales.

\section{Materials and Methods}

A prospective, descriptive study was performed of a selected sample of cases in which 5 patients, between 18 and 51 years, were included with ARP (4 patients with HIV co-infection with HPV and 1 HIV-negative patient with HPV infection [control Patient]). They were referred to the consultation of the Coloproctology Unit of the University Hospital of Caracas and were scheduled, in surgical plans, for rectoscopy and biopsy $(\mathrm{Bx})$ procedures, on an outpatient basis, during the period, March 1 to May 30, 2016.

\section{Inclusion criteria}

- Patients with a diagnosis of HIV / AIDS infection and anorectal pathologies.

- Patients between 18 and 51 years old.

- Patients receiving HAART with adherence to treatment.

- Viral Load (Undetectable $<20$ copies $/ \mathrm{mL}$ )

- Acceptance of the study by the patient.

\section{Exclusion criteria}

- Thrombocytopenia.

- Alterations of the PT and PTT.

- Neutropenia. 
- Commitment of the general state.

- Patients receiving treatment with acetyl salicylic acid and/or anticoagulants.

- Viral load and control lymphocyte subpopulation $>5$ months.

After signing an informed consent and after prepositioning of rectal enema (Fleet enema ${ }^{\circledast}$ ) by the patient they underwent individually Bx of RM. Prior to the procedure the patient was placed in knee-chest position for anal inspection and then cleaning the area with soap solution of betadine. Then sample collection of $\mathrm{RM}$ was performed through rectal anoscope, with a special Bx clip, after placement of a local anesthetic (Lidocaine 2\%). All patients were instructed to get antibiotic prophylaxis before the study (oral Metronidazole and ciprofloxacin). RM samples were properly identified in each patient. They were placed in a sterile plastic bottle with $1 \mathrm{~mL}$ of $2 \%$ glutaraldehyde for subsequent histological processing. Later samples were immediately transported for routine processing for Transmission Electron Microscopy (TEM). They were fixed with Karnovsky solution with Millonig phosphate buffer ( $\mathrm{pH} 7.4$ and $320 \mathrm{mOsm}$ ) and post-fixed with $\mathrm{OsO}_{4}$ under the same conditions of $\mathrm{pH}$ and osmolarity. After dehydration was applied in increasing concentrations of ethanol and inclusion in epoxy resin. Thin sections (60 - $90 \mathrm{~nm}$ ) were obtained with a Porter-Blum MT2-B ultramicrotome. Grids were examined in a Transmission Electron Microscope JEM-1011 $(80 \mathrm{kV})$, and Tecnai G-2 Spirit Biotwin (120 kV).

In the execution of the present investigation, several factors that, in some way, limited the study were present: 1) Cost of the materials (required for the processing of the samples). 2) Need for specialized personnel. 3) Transport of samples (samples had to be transferred to 2 different sites, which were not located within the institution (HUC). 4) Lack of updated data (Not all patients had viral load and lymphocyte subpopulation of recent data).

Ethics Statement: The use of rectal mucosa of HIV/AIDS patients was approved by the Ethical Committee of the University Hospital of Caracas. A written consent was obtained from patients.

\section{Results}

The characteristics of the patients who participated in the study are described in the following table:

\begin{tabular}{ll}
\hline \multicolumn{1}{c}{ FEATURES OF PATIENT WITH HIV/AIDS $(\mathrm{n}=4)$} & \\
\hline AGE & $28.4(21-48)$ years \\
SEX & Male \\
HAART & $\mathrm{n}=4$ \\
SMOKING HABITS & $\mathrm{n}=4$ \\
HISTORY OF RECEPTIVE ANAL SEX (MSM) & $\mathrm{n}=4$ \\
CD4 CELL COUNTS: & \\
$<200$ cell/mm $\mathrm{mm}^{3}$ & $\mathrm{n}=1$ \\
$200-499 \mathrm{cell}^{2} / \mathrm{mm}^{3}$ & $\mathrm{n}=2$ \\
$>500 \mathrm{cell} / \mathrm{mm}^{3}$ & $\mathrm{n}=1$
\end{tabular}


VIRAL LOAD (Undetectable $<20$ copies $/ \mathrm{mL}$ )

HISTORY OF RECEPTIVE ANAL SEX

NUMBER OF SEXUAL PARTNERS

CONSUMPTION OF DRUGS

ANAL CYTOLOGY PRIOR TO THE STUDY

ANAL HPV GENOTYPE DTECTED PRIOR TO THE STUDY (Cytology)

(High risk: 16,18, 31, 33, 39, 45, 51, 52, 53, 56, 58, 59, 68, 73 y 82)

(Low risk: 6, 11, 40, 42, 43, 44, 53, 54, 61, 72, 73 y 81 )

ANORECTAL PATHOLOGY

SYMPTOM

FEATURES OF PATIENT CONTROL (HIV negative) $\mathrm{n}=1$

AGE

SEX

SMOKING HABITS

SEXUAL IDENTITY

CONSUMPTION OF DRUGS

NUMBER OF SEXUAL PARTNERS

HISTORY OF RECEPTIVE ANAL SEX

ANAL CYTOLOGY PRIOR TO THE STUDY

ANAL HPV GENOTYPE DTECTED PRIOR TO THE STUDY (Cytology)

(High risk: 16,18, 31, 33, 39, 45, 51, 52, 53, 56, 58, 59, 68, 73 y 82)

(Low risk: 6, 11, 40, 42, 43, 44, 53, 54, 61, 72, 73 y 81)

ANORECTAL PATHOLOGY

SYMPTOM

$$
\begin{aligned}
& \mathrm{n}=4 \\
& \mathrm{n}=4 \\
& \geq 4 \\
& \mathrm{n}=0
\end{aligned}
$$

Only one patient

had anal squamous

intraepithelial lesion

low grade (L-ASIL)

Genotype $33(\mathrm{n}=1)$

More than one type: 33 y $6(n=1)$

Nontypeable $(\mathrm{n}=2)$

Anal Fistula $(\mathrm{n}=1)$

Anal Fissure $(\mathrm{n}=1)$

Anal Warts $(\mathrm{n}=2)$

Secretion by Recto $(\mathrm{n}=3)$

Asymptomatic ( $\mathrm{n}=1)$

55 years

Male

Present

Heterosexual

no

2

no

Normal

Genotype 53

Anal Warts

Asymptomatic

The following results showing ultrastructural pathological findings in rectal and muscular mucosa of patients with HIV/AIDS and anorectal pathologies at micrometric and at nanometric scales:

Ultrastructural pathological findings, at $10^{-6}$ scale evidenced: 1) Intestinal mucosa: vacuoles of mucus of different sizes that seem to be fused (Figure 1). 2) Smooth muscle cells: loss of definition of contractile myofilaments mass (Figure 2). 3) Unmyelinated axons and terminals of Schwann cells: Edema and loss of their plasma membranes in some areas of association with axon terminals as well as abundant collagen fibers associated with Schwann cells (Figure 3). 
The following figures produced during this research show the ultrastructural findings found (Figures 1-9).

On the other hand, findings, at $10^{-9}$ scale evidenced: 1) Smooth muscle cells: folded wrapper cores and edema of mitochondria and rough endoplasmic reticulum cisterns (RER) (Figure 4). 2) Unmyelinated axon terminals: Loss of synaptic vesicles (Figure 5). 3) Fibroblasts: One observes mitochondria and cisterns of RER with alterations (Figure 6).4) Swelling of mitochondria and cristae

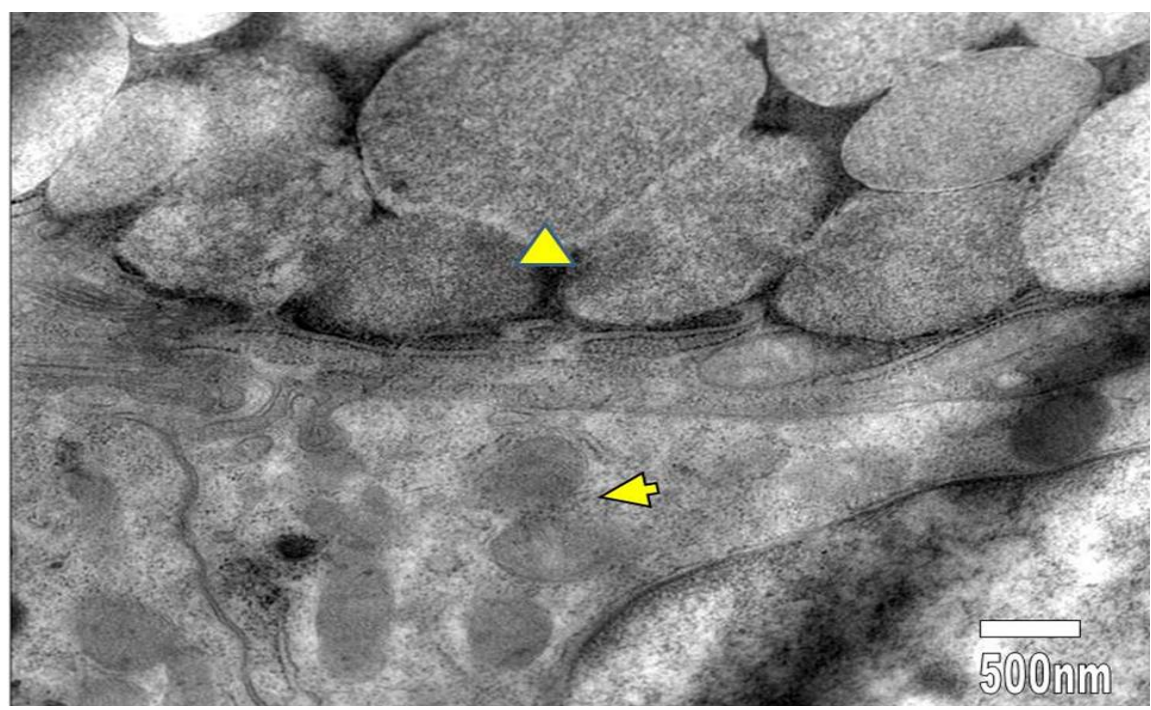

Figure 1. Intestinal mucosa: vacuoles of mucus of different sizes that seem to be fused (triangle). Normal appearance of mitochondria in some areas (arrowheads).

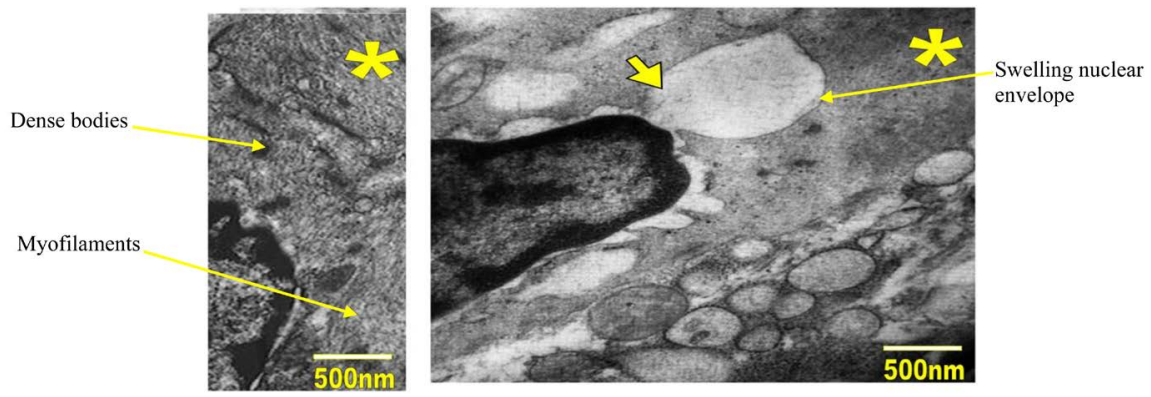

Figure 2. Loosing of myofilaments and dense bodies

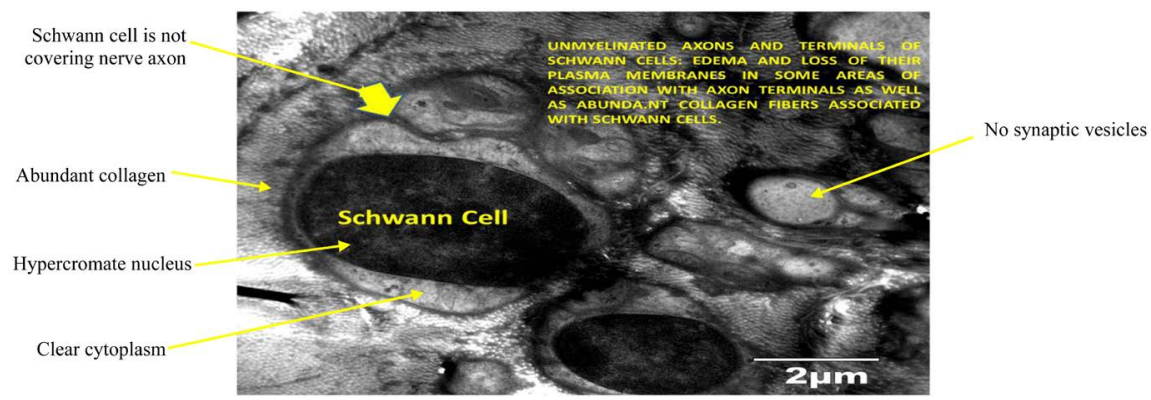

Figure 3. Retraction of the Schwann cells and loosing of the envelope. Axons are not covered by the Schwann cells. 

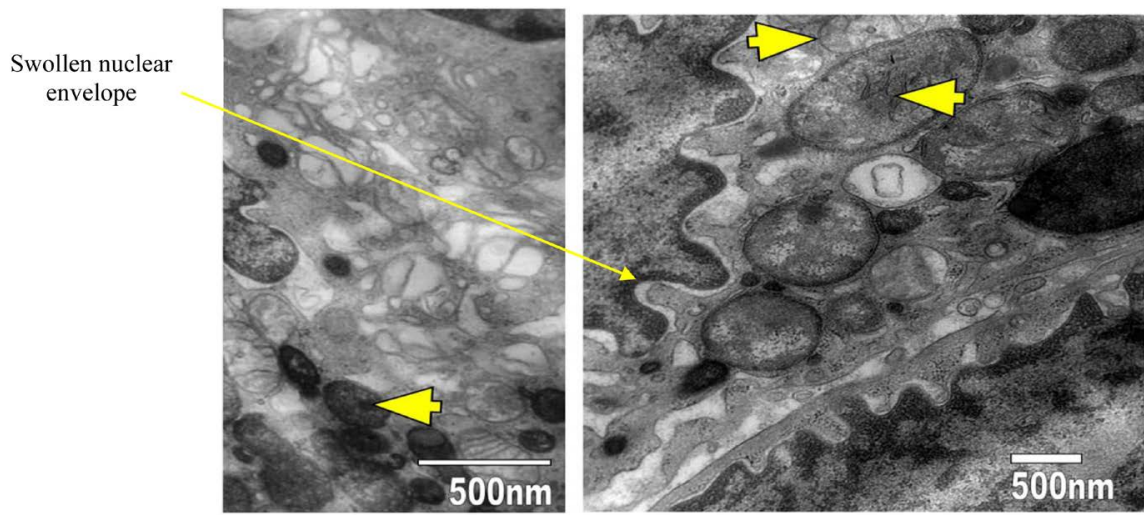

Figure 4. Smooth muscle cell of mucosa with swelling of mitochondria.
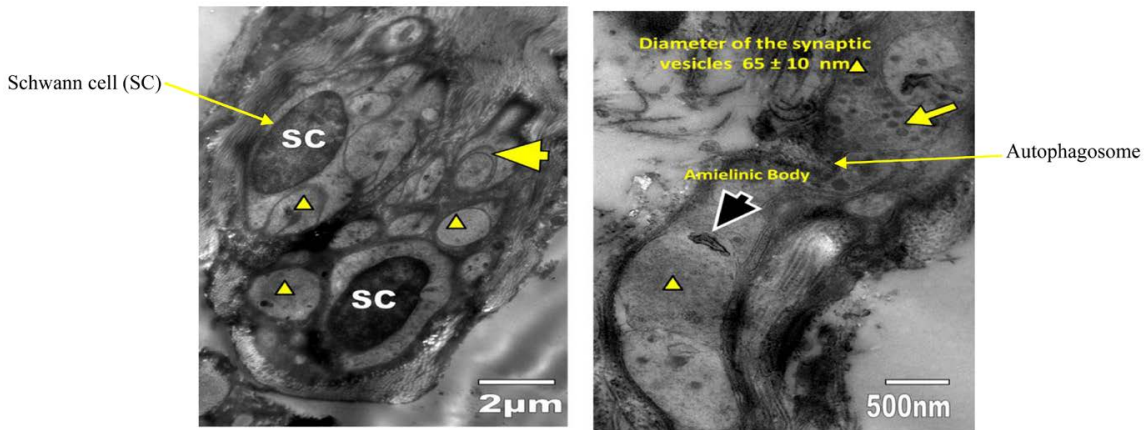

Figure 5. Terminal of the unmyelinated axons: Loss of synaptic vesicles/neurotransmitters (triangle).
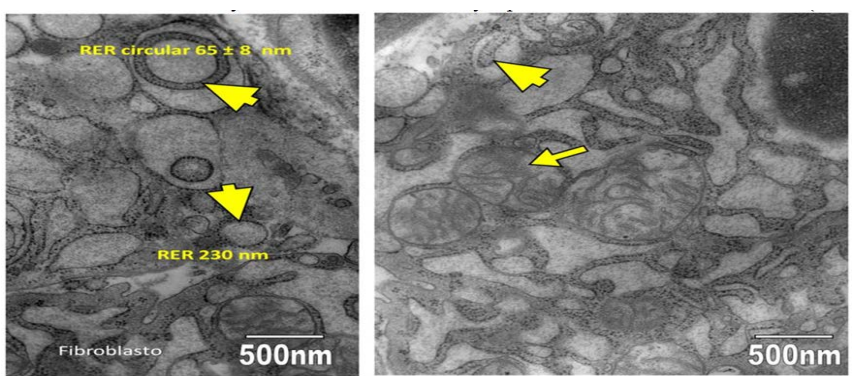

Figure 6. Fibroblast: Mitochondria and cisterns of RER swollen.

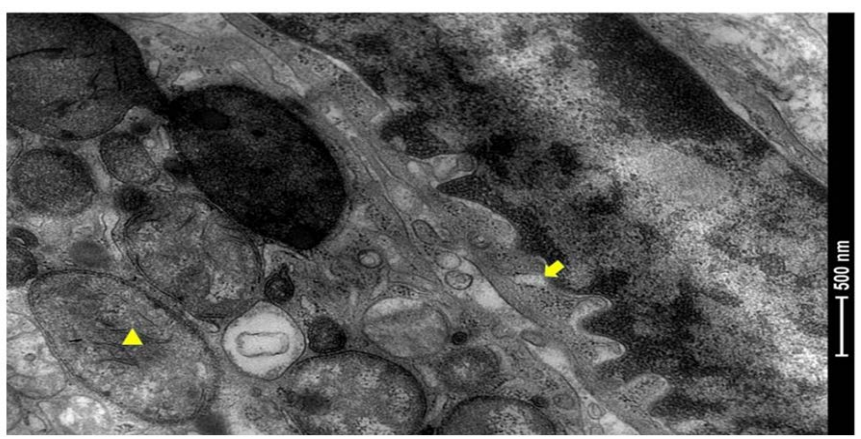

Figure 7. The triangle above shows paracrystalline inclusions that are formed starting from the cytochromes of the internal membrane when the integrity of the mitochondrial cristae are lost. The arrow shows the swollen nuclear envelope. 


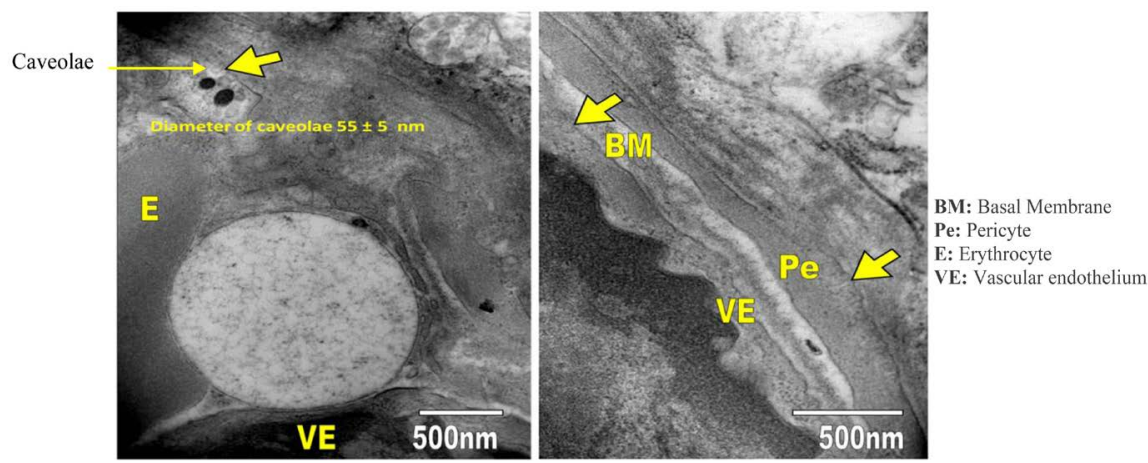

Figure 8. Small capillaries. Membrane of the endothelial cell with decreasing of caveolae.

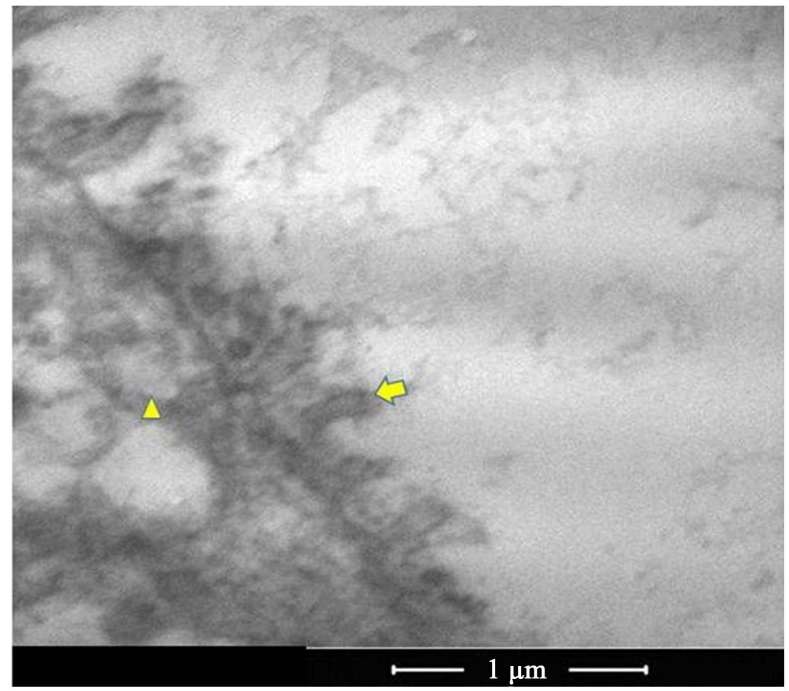

Figure 9. The electronic micrograph of the control patient (HIV negative) shows enterocyte hairiness (arrowheads) and a vacuolated cytoplasm (triangle).

(Figure 7). 5) Membrane of the endothelial cell with decreasing of caveolae (Figure 8).

\section{Relevant ultrastructural alterations at nanoscale}

1) Smooth muscular cells: Loss of myofilaments and dense bodies which may produces alteration of the contractile function of the smooth anorectal muscle.

2) Unmyelinated axon terminal: Loss of synaptic vesicles/neurotransmitters which may produce alteration of peristalsis and contraction of smooth muscle.

3) Swelling of nuclear envelopes, Golgi and RER: This may produce alteration in completing the transport, and synthesis of proteins.

4) Swelling of mitochondria and cristae: This may produce alteration of their functions (Krebs cycle, chain of electron transport, oxidative phosphorylation and ATP production).

5) Small capillaries: Membrane of the endothelial cell with decreasing or absence of Caveolae.

In sum, the pathological findings of the rectal and muscle mucosa such as in- 
flammation (nuclear envelopes and cytoplasmic organelles), nervous fiber absence of synaptic vesicles in unmyelinated axons and muscular smooth cells with loosing of myofilaments and dense bodies can produce alteration of the contractile function, intestinal and anorectal dysfunction.

\section{Discussion}

Studies in patients with HIV co-infection with HPV showed a high prevalence of HPV anorectal infection and Squamous Intraepithelial Lesion (SIL) in MSM; the incidence of high-grade squamous intraepithelial lesions (HSIL) was higher in patients receiving HAART. It is believed that this is due to increased expression of HPV secondary to the interaction between the TAT gene of HIV-1 and the p97 protein which prevents the repression of gene E2 of HPV. SIL progression to invasive cancer requires several years, due to prolonging survival with HAART, paradoxically involved in the increased risk of anal cancer [20] [21] [22] [23]. In this research only one patient had anal squamous intraepithelial lesion, low grade (L-ASIL).

Studies in patients with HIV/AIDS have reported inflammation of the rectal mucosa (Proctitis) [1] [7] [8]. In this specific study it was evidenced, at the ultrastructural level, inflammation of nuclear envelopes and cytoplasmic organelles (mitochondria and RER) as well as edema in some areas of association with axon terminals.

Pérez-Matute et al. stated that: "Several factors can affect the functionality of the mitochondria, (inter alia), aging, infections or certain medications used to treat HIV/AIDS. Nucleotide reverse transcriptase inhibitors (NRTIs) have been considered as the main source of mitochondrial toxicity related to HAART, since these can inhibit Pol- $\gamma$, the DNA polymerase responsible for the synthesis of mitochondrial DNA, although non-nucleoside transcriptase inhibitors (NNRTI) or protease inhibitors (PI) they have also shown mitochondrial toxicity" (Pérez-Matute $\mathrm{P}$ et al., 2013) [24]. In this study, the following findings were found, via electron microscopy, at the level of the mitochondria, namely, swelling of mitochondria at the level of smooth muscle cell of mucosa and paracrystalline inclusions that are formed starting from the cytochromes of the internal membrane when the integrity of the mitochondrial cristae are lost.

Mergia Ayalew (2017) [25], described that:

Caveolae structures are present in abundance in mechanically stressed cells such as endothelial cells and adipocytes. Caveolin 1 (Cav-1) is a major component of the caveolae structure and it is expressed in a variety of cell types including macrophages, which are susceptible to human immunodeficiency virus (HIV) infection. Since Cav-1 and caveolae are abundant and play essential roles in endothelial cell function, there has to be link with HIV induced endothelial dysfunction. The influence of HIV on Cav-1 redistribution and cholesterol efflux can alter cholesterol homeostasis and membrane molecule compositions which can impact caveolae/Cav-1 func- 
tions and the caveolae structure. In addition, multiple studies suggest that antiretroviral drugs induce reactive oxygen species (ROS) production in HIV infected individuals. Thus, up-regulation of the multifunctional Cav-1 by elevated ROS production as a consequence of HIV infection and continuous anti-HIV drug treatments may result in premature cellular senescence accumulation overtime, subsequently causing cellular dysfunction and pathology.

In this research the following was evidenced, at the ultrastructural level, in small capillaries: Membrane of the endothelial cell with decreasing of Caveolae and in some areas their absence, which may produces alteration of the cellular processes depending of Caveolae and/or Caveolins.

Edagwa et al. affirmed that: "Overwhelming evidence supports the notion that cells and tissues sheltered from the lymphoid system, such as the central nervous system, lymph nodes, testes, gut, vaginal epithelium and lungs, serve as viral sanctuaries that complicate HIV treatment. This occurs, in part, because many drugs cannot infiltrate these sites in sufficient concentrations to exert optimal therapeutic responses. The HIV continues to replicate in anatomical and intracellular sites where antiretroviral therapy (ART) has restricted access. Penetration of ART into viral sanctuaries is limited by blood tissue barriers. Delivery of ART to these sites will have a great impact on HIV eradication efforts".... Lymphoid tissues and gut are considered the major reservoirs for HIV replication; therefore targeting antiretroviral therapies to these areas is crucial to HIV eradication. (Edagwa Benson J. et al., 2014) [26]. Corsi et al. stated that: "Certainly, further research is required to better define the antiretroviral activity once the nano-drug has passed through the barrier...Interestingly, the nanoparticles were able to overcome P-gp-mediated efflux from bowel mucosa, allowing caveolaeand clathrin-mediated transcytosis of the drug, with subsequent absorption. However, although potentially significant, antiretroviral drugs nanoformulations to specifically overcome P-gp activity still remain poorly investigated" (Corsi Fabio et al., 2016) [27].

Wang Zhenjia et al. (2011) [28], described that:

The transcellular pathway is the dominant pathway of transporting macromolecules in endothelial cells. The caveolae is the major vesicular transporter in endothelial cells, occupying as much as $70 \%$ of the endothelial membrane in blood capillaries, and selectively macromolecular endocytoses. Across the cells (such as, their size $>3 \mathrm{~nm}$ ), Caveolae are membrane invaginations $50-100 \mathrm{~nm}$ in diameter that pinch off to form intracellular vesicles and can move macromolecules across the endothelial barrier.

Beloqui et al. argued that: "The non-phagocytic pathways, involving clathrin-mediated endocytosis, caveolae-mediated endocytosis and macropinocytosis, are the most common mechanisms of nanoparticle absorption/transcytosis by the oral route" (Beloqui Ana et al., 2013) [29]. In this research we observed that 
in patients with HIV/AIDS and anorectal pathologies there is alteration of the endothelial cell membrane of small capillaries with decrease of caveolae and in some areas their absence. Thus we ask ourselves whether the pathway mediated by caveolae would be or would not be an optimal strategy to apply therapeutic nanoparticles through the endothelial barrier in patients with HIV/AIDS. It is important to point out the small attention given to molecular pathways which control the transport of drugs to the tissue.

It is evident that there are many challenges to research which will allow a better understanding of what takes place at the molecular level of the IM. This is with the objective of uncovering the target that could optimize the penetration of nano-drugs able to eradicate HIV in those sites of the intestine where conventional antiretroviral have restricted access. Another of the challenges is to achieve that nano-ART could pass the regulatory barrier expeditiously, demonstrating its efficacy, safety and a good balance risk/benefit for its approval and could be available to the medical community in general.

\section{Conclusions}

These results point to the fact that HIV causes visible changes, at micrometric and nanometric scales, in the rectal mucosa despite the fact that HAART has achieved complete suppression of viral load in peripheral blood. All these above captioned alterations can generate intestinal and anorectal dysfunction in these patients. The decreasing of Caveolae or their absence in some areas in the membrane of the endothelial cell may produce alteration of the cellular processes depending of Caveolae and/or Caveolins. Nano-drug could play an important role in drug delivery in sites where conventional ART have limited access, so as to achieve sufficient concentrations to execute optimal therapeutic responses and virus eradication in anatomical and intracellular sites.

\section{References}

[1] Annunziato, M.A. (1997) Clinical, Mycotic, and Anorectal Immunohistochemically Findings in Patients with AIDS. University Hospital. Dr. Angel Larralde, University of Carabobo, Valencia, Venezuela. Department of Internal Medicine. Special Research to Qualify for the Title of Internal Medicine Specialist.

[2] Smith, P.D., et al. (1992) Gastrointestinal Infections in AIDS. Annals of Internal Medicine, 116, 63-77. https://doi.org/10.7326/0003-4819-116-1-63

[3] Gold, J.W., Telzak, E.E. and White, D.A. (1996) Management of the HIV-Infected Patient, Part I. The Medical Clinics of North America, 80, 1223-1238, 1395-1414.

[4] Carvajal, A. (1997) Acquired Immunodeficiency Syndrome. In: Núñez, M.J. and Gómez, M.J., Eds., Medical Microbiology, 2 Edition, Vice-Rectorate Academic U.C.V, Venezuela, 885-913.

[5] Brar, H.S., et al. (1998) Anorectal Pathology in AIDS. Gastrointestinal Endoscopy Clinics of North America, 8, 913-931.

[6] Kent, C., Samuel, M. and Winskelstein, W.J.R. (1987) The Role of Anal/Genital Warts in HIV Infection. JAMA, 258, 3385-3386.

https://doi.org/10.1001/jama.1987.03400230045017 
[7] Annunziato, M.A. (2000) Anorectal Findings in Patients Infected with HIV. University Hospital of Caracas, Central University of Venezuela, Caracas, Venezuela. Special Research to Qualify for the Title of Specialist on Infectious Diseases.

[8] Orkin, B.A. and Smith, L.E. (1992) Perianal Manifestations of HIV Infection. Diseases of the Colon \& Rectum, 35, 310-314. https://doi.org/10.1007/BF02048106

[9] Guadalupe, et al. (2003) Severe $\mathrm{CD}^{+}{ }^{+}$t-Cell Depletion in Gut Lymphoid Tissue during Primary Human Immunodeficiency Virus Type 1 Infection and Substantial Delay in Restoration Following Highly Active Antiretroviral Therapy. Journal of Virology, 80, 11708-11717. https://doi.org/10.1128/JVI.77.21.11708-11717.2003

[10] Belmonte, L., et al. (2007) The Intestinal Mucosa as a Reservoir of HIV-1 after Successful HAART. AIDS, 21, 2106-2108. https://doi.org/10.1097/QAD.0b013e3282efb74b

[11] Mariangela, C., et al. (2013) R5 HIV-1 Envelope Attracts Dendritic Cells to Cross the Human Intestinal Epithelium and Sample Luminal Virions via Engagement of the CCR5. EMBO Molecular Medicine, 5, 776-794. https://doi.org/10.1002/emmm.201202232

[12] Veazey, R.S. and Lackner, A.A. (1998) The Gastrointestinal Tract and the Pathogenesis of AIDS. AIDS, 12, S35-S42.

[13] Scheider, T., Ullrich, R. and Zeitz, M. (1997) Immunopathology of Human Immunodeficiency Virus Infection in the Gatrointestinal Tract. Seminars in Immunopathology, 18, 515-533. https://doi.org/10.1007/BF00824056

[14] Guadalupe, et al. (2006) Viral Suppression and Immune Restoration in the Gastrointestinal Mucosa of Human Immunodeficiency Virus Type 1-Infected Patients Initiating Therapy during Primary or Chronic Infection. Journal of Virology, 80, 8236-8247. https://doi.org/10.1128/JVI.00120-06

[15] Sanjay, S., et al. (2004) P Glycoprotein in Human Immunodeficiency Virus Type 1 Infection and Therapy. Antimicrobial Agents and Chemotherapy, 48, 1073-1108. https://doi.org/10.1128/AAC.48.4.1073-1081.2004

[16] Kotler, D.P. (1989) Intestinal and Hepatic Manifestations of AIDS. Advances in Internal Medicine, 34, 43-72.

[17] Greenson, J.K., Belitsos, P.C., Yardley, J.H., et al. (1991) AIDS Entheropathy: Occult Enteric Infections and Duodenal Mucosal Alterations in Chronic Diarrhea. Annals of Internal Medicine, 114, 366-372. https://doi.org/10.7326/0003-4819-114-5-366

[18] Kottler, D.P., Reka, S. and Clayton, F. (1993) Intestinal Mucosal Inflamation Associated with Human Immunodeficiency Virus Infection. Digestive Diseases Sciences, 38, 1119-1127. https://doi.org/10.1007/BF01295730

[19] Paiardini, M., et al. (2008) Mucosal Immune Dysfunction in AIDS Pathogenesis. AIDS Reviews, 10, 36-46.

[20] Fox, A., et al. (2005) The Value of Anal Cytology and Human Papillomavirus Typing in the Detection of Anal Intraepithelial Neoplasia: A Review of Cases from an Anoscopy Clinic. Sexually Transmitted Infections, 81, 142-146. https://doi.org/10.1136/sti.2003.008318

[21] Piketty, C., et al. (2008) Marked Increase in the Incidence of Invasive anal Cancer among HIV-Infected Patients Despite Treatment with Combination Antiretroviral Therapy. AIDS, 19, 1203-1211.

[22] Palefky, J.M. (1999) Anal Squamos Intraepitelial: Relation to HIV and Human Papillomavirus Infection. Journal of AIDS, 21, S42-S48.

[23] Barbaro, G. and Barbarini, G. (2007) HIV Infection and Cancer in the Era of Highly 
Active Antiretroviral Therapy (Review). Oncology Reports, 17, 1121-1126. https://www.ncbi.nlm.nih.gov/pubmed/17390054

[24] Pérez-Matute, P., et al. (2013) Role of Mitochondria in HIV Infection and Associated Metabolic Disorders: Focus on Nonalcoholic Fatty Liver Disease and Lipodystrophy Syndrome. Oxidative Medicine and Cellular Longevity, 2013, Article ID: 493413. https://www.hindawi.com/journals/omcl/2013/493413/

[25] Mergia, A. (2017) The Role of Caveolin 1 in HIV Infection and Pathogenesis. Viruses, 9, 129. http://www.mdpi.com/1999-4915/9/6/129 https://doi.org/10.3390/v9060129

[26] Benson, E.J., et al. (2014) Development of HIV Reservoir Targeted Long Acting Nanoformulated Antiretroviral Therapies. Current Medicinal Chemistry, 21, 4186-4198. https://www.ncbi.nlm.nih.gov/pmc/articles/PMC4281174/ https://doi.org/10.2174/0929867321666140826114135

[27] Corsi, F., et al. (2016) Antiretroviral Therapy through Barriers: A Prominent Role for Nanotechnology in HIV-1 Eradication from Sanctuaries. Journal of Pharmacy and Pharmacology, 4, 328-339.

https://air.unimi.it/retrieve/handle/2434/526744/911463/7-JPP2016021701.pdf

[28] Wang, Z., et al. (2011) Targeting of Nanoparticle-Complexed Drugs across the Vascular Endothelial Barrier via Caveolae. IUBMB Life, 63, 659-667.

https://www.ncbi.nlm.nih.gov/pmc/articles/PMC3142311/ https://doi.org/10.1002/iub.485

[29] Beloqui, A., et al. (2013) Mechanism of Transport of Saquinavir-Loaded Nanostructured Lipid Carriers across the Intestinal Barrier. Journal of Controlled Release, 166, 115-123. https://doi.org/10.1016/j.jconrel.2012.12.021

https://cdn.uclouvain.be/public/Exports\%20reddot/ir-ldri/images/Beloqui2013.pdf 